\title{
Viscoelasticity and Birefringence of Low Birefringent Polyesters
}

\author{
Tadashi Inoue, ${ }^{\dagger}$ Kazuyuki FuJIWARA, Deug-Soo RYU, Kunihiro OSAKI, \\ Michiaki FUJI, ${ }^{*}$ and Kazuo SAKURAI* \\ Institute for Chemical Research, Kyoto University, \\ Uji, Kyoto 611-0011, Japan \\ *O-PET Development, Kanebo Ltd., \\ 5-90, Tomobuchi, Miyakojima Osaka 534-8666, Japan
}

(Received November 8, 1999)

\begin{abstract}
Dynamic viscoelasticity and dynamic birefringence were investigated for a series of polyesters with various combinations of dimethyl decalin 2,6-carboxylate, DMHN and dimethyl terephthalate, DMT as acid component and 9,9-bis[4-(2-hydroxy-ethoxy)phenyl]fluorene, BPEF and ethylene glycol, EG as diol component. The lowering of the stress-optical coefficient was achieved by the displacement of phenylene (DMT) with a hydrogenated naphthylene ring (DMHN) in the main chain and by the introduction of BPFE in the place of EG. The former choice lowered the intrinsic birefringence but did not affect the chain flexibility. The latter enhanced the flexibility but did not affect the intrinsic birefringence. The systematic result clearly revealed the importance of two different factors, the anisotropy of structural units and the chain flexibility, in molecular designs for polymers with low stress-optical coefficient. KEY WORDS Intrinsic Birefringence / Stress-Optical Coefficient / Polyester /
\end{abstract}

The birefringence of a polymeric material is often evaluated by measuring the remaining birefringence of a specimen prepared with the method similar to polymer processing, injection molding for example. This may be good for the evaluation of a particular material. On the other hand, it is not appropriate for comparing different species of polymers. The remaining birefringence depends on the rheological properties and thus varies much with processing conditions. For example, the result depends much on the molecular weight in addition to the properties characteristic of the chemical species, so that the evaluation must be performed for various molecular weights or else at various processing conditions.

Recently the authors have studied the dynamic birefringence, the birefringence under oscillatory deformation, and revealed the details of the relaxation process of the birefringence and its relation to the orientation of the structural unit. ${ }^{1-4}$ This method allowed us the comparison of birefringence of polymers of different species of any molecular weights. The origin of birefringence in various states, various temperatures for example, has been clarified for vinyl polymers through systematic studies.

The use of a low birefringent material is one of the ideas for removing the harmful effect of the birefringence in optical use of polymeric materials. Monomers of low anisotropy such as cyclic olefins lead to polymers with low birefringence. ${ }^{5,6}$ Low birefringence may also be achieved through mixing of materials with birefringence of opposite signs. ${ }^{7}$ Copolymerization of monomers with opposite anisotropies would improve the uniform mixing and still have the same effect for lowering of birefringence. ${ }^{8}$ The authors have been studying the possibility in polyesters with various combinations of components and have obtained a series of copolyesters with consider-

\footnotetext{
${ }^{\dagger}$ To whom correspondence should be addressed.
}

ably low birefringence. ${ }^{9}$ The purpose of the present study is to clarify the mechanism of lowering of the birefringence through detailed examination of the dynamic birefringence.

\section{MATERIALS AND METHOD}

\section{Materials}

The test materials were a series of copolyesters with various combinations of dimethyl decalin 2,6-carboxylate, DMHN and dimethyl terephthalate, DMT as acid component and 9,9-bis[4-(2-hydroxy-ethoxy)phenyl]fluorene, BPEF and ethylene gricol, EG as diol component. The preparation of the materials was published elsewhere. ${ }^{9}$ The structures of the components are shown in Figure 1. The compositions of the polymers used in the present study are shown in Table I together with the molecular weights, $M_{W}$, determined by GPC measurement and the glass transition temperature, $T_{\mathrm{g}}$, determined by DSC measurement.

The DMHN was employed to represent a saturated cy-

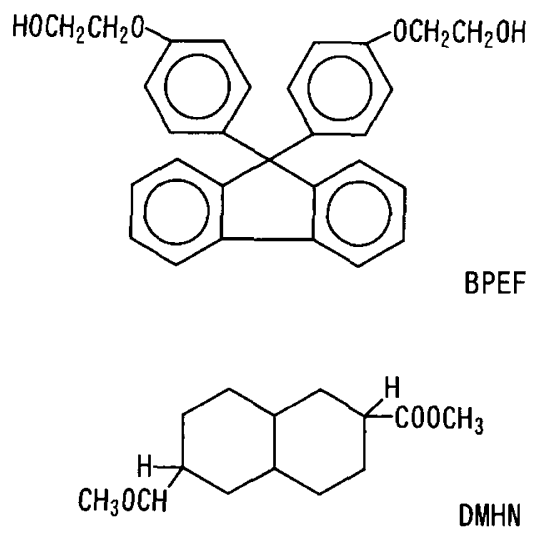

Figure 1. The components of the samples. 
Table I. Composition, molecular weight, and glass transition temperature of samples

\begin{tabular}{|c|c|c|c|c|c|c|}
\hline \multirow{2}{*}{$\begin{array}{l}\text { Polymer } \\
\text { code }\end{array}$} & \multicolumn{2}{|c|}{ Acid $/ \mathrm{mol}^{2}{ }^{\mathrm{a}}$} & \multicolumn{2}{|c|}{$\mathrm{Diol} / \mathrm{mol} \%^{\mathrm{a}}$} & \multirow{2}{*}{$M_{w}{ }^{\mathrm{b}}$} & \multirow{2}{*}{$T_{\mathrm{g}}{ }^{\mathrm{c}}$} \\
\hline & DHMN & DMT & BREF & $\mathrm{EG}$ & & \\
\hline TF40 & 0 & 100 & 40 & 60 & 44000 & 125 \\
\hline TF70 & 0 & 100 & 70 & 30 & 41000 & 142 \\
\hline HNF20 & 100 & 0 & 20 & 80 & 88000 & 85 \\
\hline HNF40 & 100 & 0 & 40 & 60 & 39000 & 108 \\
\hline HNF70 & 100 & 0 & 70 & 30 & 49000 & 129 \\
\hline HN50F70 & 50 & 50 & 70 & 30 & 47000 & 135 \\
\hline HN40F70 & 40 & 60 & 70 & 30 & 47000 & 135 \\
\hline HN20F70 & 20 & 80 & 70 & 30 & 43000 & 139 \\
\hline
\end{tabular}

${ }^{a}$ Determined by NMR. ${ }^{b}$ Determined by GPC calibrated with polystyrene standards. ${ }^{\mathrm{c}}$ Determined by DSC (heating rate $=10 \mathrm{~K}$ $\min ^{-1}$ ).

clic structure in the main chain and was expected to be a unit with low anisotropy compared to aromatic rings in the main chain. The BPEF was initially supposed to be a unit with negative birefringence (which turned out to be wrong in the present study) and expected to compensate the positive value due to other components.

Films with $0.3 \mathrm{~mm}$ thickness were made by melt press method at $200^{\circ} \mathrm{C}$.

\section{Measurements}

Details of the apparatus and its operation for dynamic birefringence measurements were described elsewhere. ${ }^{10}$ The complex Young's modulus $E^{*}(\omega)=E^{\prime}(\omega)+$ $\mathrm{i} E^{\prime \prime}(\omega)$ and the complex strain-optical ratio $O^{*}(\omega)=$ $O^{\prime}(\omega)+\mathrm{i} O^{\prime \prime}(\omega)$ were measured with an elongational oscillatory rheometer (DVE III, Reoroji Co. Ltd., Kyoto) equipped with an optical system. Here the strain-optical ratio is the ratio of the birefringence to the strain in oscillatory deformation; $O^{\prime}(\omega)$ and $O^{\prime \prime}(\omega)$ respectively represent the in-phase and out-of-phase components of the birefringence just as $E^{\prime}(\omega)$ and $E^{\prime \prime}(\omega)$ represent the in-phase and out-of-phase components of the stress. The range of frequency was from 1 to $130 \mathrm{~Hz}$. The measurements were performed at several temperatures between $T_{\mathrm{g}}+20^{\circ} \mathrm{C}$ and $T_{\mathrm{g}}$.

\section{Method of Analysis}

The data were analyzed with the modified stressoptical rule (MSOR). ${ }^{10}$ This is to assume that $E^{*}(\omega)$ and $O^{*}(\omega)$ are written as follows.

$$
\begin{gathered}
E^{*}(\omega)=E^{*}{ }_{\mathrm{R}}(\omega)+E_{\mathrm{G}}^{*}(\omega) \\
O^{*}(\omega)=C_{\mathrm{R}} E^{*}{ }_{\mathrm{R}}(\omega)+C_{\mathrm{G}} E^{*}{ }_{\mathrm{G}}(\omega)
\end{gathered}
$$

The MSOR states that the complex modulus is composed of two components, $E^{*}{ }_{\mathrm{R}}(\omega)$ and $E^{*}{ }_{\mathrm{G}}(\omega)$, and the stressoptical ratio can also be related to two components. The subscripts $R$ and $G$ respectively represent the rubbery and the glassy component. The former is associated with longer relaxation times than the latter.

The coefficient, $C_{\mathrm{R}}$, is the stress-optical coefficient determined in the terminal flow or rubbery zones. In the present study, the lowest frequency belonged to the boundary between the rubbery and the glass-to-rubber transition zones. The stress-optical coefficient could be derived as the ratio $O^{\prime}(\omega) / E^{\prime}(\omega)$ in this region accord- ing to the results for various amorphous polymers.

The coefficient $C_{\mathrm{G}}$ is defined as the ratio $O^{\prime \prime}(\omega) / E^{\prime \prime}$ $(\omega)$ in the glassy zone. The ratio remains constant over a wide frequency range for many polymers. This implies that the stress relaxation is due solely to the G component of stress and the $\mathrm{R}$ component of stress does not relax in the relevant time range. The ratio $O^{\prime \prime}(\omega) / E^{\prime \prime}(\omega)$ was constant in the glassy zone for the present systems and was taken as $C_{\mathrm{G}}$.

The frequency dependence of the $\mathrm{R}$ component modulus, $E^{*}{ }_{\mathrm{R}}(\omega)$, is well described with the bead-spring model of Rouse and the molecular weight of the Rouse segment, $M_{\mathrm{s}}$, is estimated from the high frequency limit of the modulus, $E_{\mathrm{R}}^{\prime}(\omega)$.

$$
M_{\mathrm{s}}=3 \rho R T / E_{\mathrm{R}}^{\prime}(\infty)
$$

The $M_{\mathrm{s}}$ thus determined is approximately equal to the molecular weight of the Kuhn statistical segment determined from the dilute solution studies for many polymers. ${ }^{11}$ The $M_{\mathrm{s}}$ can be a measure of the stiffness of the chain as compared to the assumed chain of free rotational bonds.

Some other characteristic parameters can be derived from the quantities obtained through the MSOR. The photo-elastic coefficient, $C_{\mathrm{d}}=O^{\prime}(\omega) / E^{\prime}(\omega)$ at the limit of high frequency, is given by

$$
C_{\mathrm{d}}=C_{\mathrm{G}}+C_{\mathrm{R}} E_{\mathrm{R}}^{\prime}(\infty) / E^{\prime}(\infty)
$$

The intrinsic birefringence is given by ${ }^{12}$

$$
\Delta n_{0}=5 C_{\mathrm{R}} E_{\mathrm{R}}^{\prime}(\infty) / 3
$$

\section{RESULTS}

\section{An Example of the Dynamic Data}

The results of $E^{*}(\omega)$ and $O^{*}(\omega)$ for the sample TF 40 is given in Figure 2. The data were obtained for temperatures, $160-121^{\circ} \mathrm{C}$ and reduced to the reference temperature $T_{\mathrm{r}}=133^{\circ} \mathrm{C}$ with the method of reduced variables. ${ }^{13}$ The master curves for $E^{\prime}(\omega)$ and $E^{\prime \prime}(\omega)$ carry typical features of the complex modulus of amorphous polymers in the glass-to-rubber transition zone and the glassy zone. The $O^{\prime}(\omega)$ and $O^{\prime \prime}(\omega)$ are always positive. The curves for $O^{\prime}(\omega)$ and $O^{\prime \prime}(\omega)$ are similar to those for $E^{\prime}(\omega)$ and $E^{\prime \prime}(\omega)$ in the following sense ; $O^{\prime}(\omega)$ is an increasing function of $\omega$ and levels off at high frequencies ; $O$ " $(\omega)$ exhibits one maximum.

For about one decade of the lowest frequencies the ratio $O^{\prime}(\omega) / E^{\prime}(\omega)$ remains approximately constant and $C_{\mathrm{R}}=1.8 \times 10^{-9} \mathrm{~Pa}^{-1}$ is obtained. For two decades of high frequencies the ratio $O^{\prime \prime}(\omega) / E^{\prime \prime}(\omega)$ is approximately constant and $C_{\mathrm{G}}=5.9 \times 10^{-11} \mathrm{~Pa}^{-1}$ is obtained.

The results for other samples were qualitatively the same as this example. The coefficients $C_{\mathrm{R}}$ and $C_{\mathrm{G}}$ were positive for all the samples tested. These are given in Table II. The results for other samples can be obtained on request.

\section{Application of the Modified Stress-Optical Rule and the Characteristic Parameters}




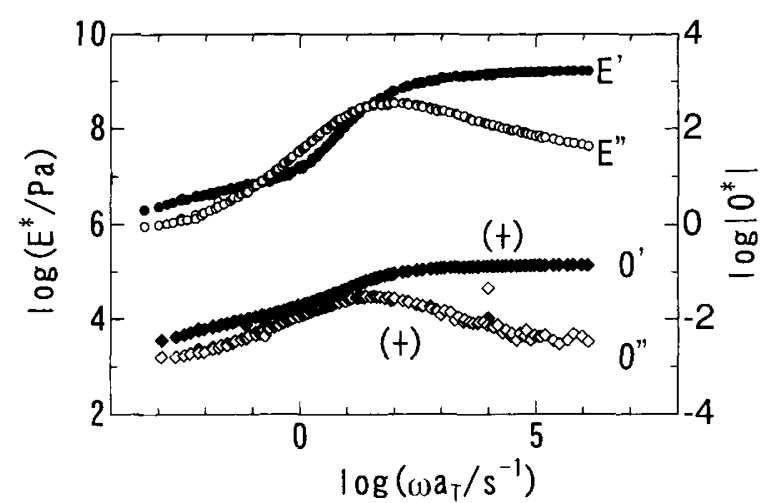

Figure 2. The complex Young's modulus and the complex strainoptical ratio for TF 40 .

Table II. Characteristic parameters for viscoelasticity and birefringence. $\mathrm{Br}=10^{-12} \mathrm{~Pa}^{-1}$

\begin{tabular}{lccccccc}
\hline $\begin{array}{c}\text { Polymer } \\
\text { code }\end{array}$ & \multicolumn{2}{c}{$C_{\mathrm{R}} / \mathrm{Br}$} & $C_{\mathrm{G}} / \mathrm{Br}$ & $C_{\mathrm{d}} / \mathrm{Br}$ & $E_{\mathrm{R}}^{\prime}(\infty) / \mathrm{MPa} E_{\mathrm{G}}^{\prime}(\infty) / \mathrm{GPa}$ & $M_{\mathrm{s}}$ & $\Delta n_{0}$ \\
\hline TF40 & 1800 & 59 & 98 & 37 & 1.6 & 340 & 0.11 \\
TF70 & 1500 & 57 & 92 & 30 & 1.2 & 450 & 0.075 \\
HNF20 & 750 & 9.5 & 27 & 25 & 1.0 & 470 & 0.031 \\
HNF40 & 450 & 17 & 29 & 39 & 1.3 & 320 & 0.029 \\
HNF70 & 323 & 21 & 34 & 55 & 1.2 & 240 & 0.030 \\
HN50F70 & 750 & 34 & 55 & 35 & 1.2 & 390 & 0.043 \\
HN40F70 & 930 & 42 & 65 & 31 & 1.1 & 430 & 0.047 \\
HN20F70 & 980 & 45 & 69 & 31 & 1.1 & 430 & 0.050 \\
\hline
\end{tabular}

The simultaneous eq 1 and 2 were solved for the component functions, $E^{*}{ }_{\mathrm{R}}(\omega)$ and $E^{*}{ }_{\mathrm{G}}(\omega)$, with the use of the data of $E^{*}(\omega)$ and $O^{*}(\omega)$ given in Figure 2 and the coefficients $C_{\mathrm{R}}$ and $C_{\mathrm{G}}$ determined by the procedure described above. The features of $E^{*}{ }_{\mathrm{R}}(\omega)$ and $E^{*}{ }_{\mathrm{G}}(\omega)$ shown in Figure 3 are qualitatively the same as those of many other amorphous polymers. The $E{ }^{*}(\omega)$, with one marked dispersion peak in the imaginary part corresponding to that of the glass-to-rubber transition, is similar to that of non-polymeric glass-forming materials. The $E{ }_{R}^{*}(\omega)$ shows a sign of rubbery plateau zone at low frequencies and a broad dispersion in the intermediate frequency region. The latter may be described with a bead-spring model of Rouse and Mooney. The molecular weight of the Rouse segment, $M_{\mathrm{s}}$, is estimated as 340 with eq 3 and $E_{\mathrm{R}}^{\prime}(\infty)=37 \mathrm{MPa}$.

Values of $E_{\mathrm{G}}^{\prime}(\infty), E_{\mathrm{R}}^{\prime}(\infty), M_{\mathrm{s}}$, and $\Delta n_{0}$ are given in Table II for all the polymers. The $E^{\prime}{ }_{G}(\infty)$ is essentially equal to the instantaneous Young's modulus, $E^{\prime}(\infty)$.

\section{DISCUSSION}

\section{Effect of Varying Acid Compositions}

For the samples TF70, HNF70, HN50F70, HN40F70, and HN20F70, the composition of the diol component is fixed at $\mathrm{BPFE}=70 \mathrm{~mol} \%$ and $\mathrm{EG}=30 \mathrm{~mol} \%$ and the composition of the acid component is varied. The variations of the characteristic parameters with the acid composition are shown in Figure 4.

The introduction of hydrogenated ring of DMHN in the main chain in the place of aromatic ring of DMT lowered all the parameters related to the birefringence: $\Delta n_{0}, C_{\mathrm{R}}$, and $C_{\mathrm{G}}$. This is in accord with the similar effect

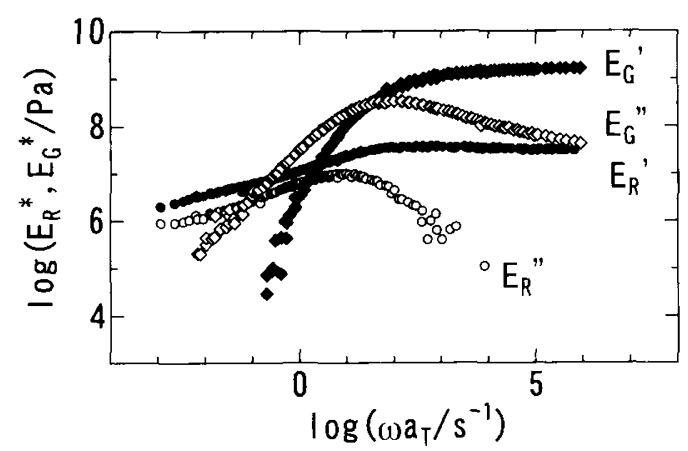

Figure 3. The component functions of the Young's modulus derived from the data of Figure 2.

attained for polyolefins with saturated rings in the main chain and probably indicates that the saturated ring in the main chain is regarded as a unit with small anisotropy. The molecular weight of the Rouse segment, $M_{\mathrm{s}}$, remained almost constant except for the HNF70, for which the acid component was solely DMHN.

\section{Effect of Varying Diol Compositions}

For the samples HNF20, HNF40, and HNF70 (HNF series), the diol compositions are varied and the acid composition is fixed at $\mathrm{DMHN}=100 \mathrm{~mol} \%$. The characteristic parameters are shown in Figure 5 . With varying fractions of $\mathrm{BPEF}$, the intrinsic birefringence, $\Delta n_{0}$, remained constant, the coefficient $C_{\mathrm{R}}$ decreased, and the coefficient $C_{\mathrm{G}}$ increased. This is in contrast with the case of varying amount of hydrogenated rings, for which all these quantities decreased with the increase of DMHN. The increase of $C_{\mathrm{G}}$ with increase of flat (or bulky) BPEF unit is in accord with previous works on various polymers. The different tendencies of $\Delta n_{0}$ and $C_{\mathrm{R}}$ may be accounted for by the variation of the chain flexibility as mentioned below.

The molecular weight of the Rouse segment, $M_{\mathrm{s}}$, decreased with increasing BPEF and therefore the chain flexibility increased. Since the stress-optical coefficient, $C_{\mathrm{R}}$, is a measure of optical anisotropy of the segments, it is proportional to $M_{\mathrm{s}}$. On the other hand, $\Delta n_{0}$ is almost constant for varying $\mathrm{BPEF}$ fraction. $\mathrm{BPEF}$ has much larger molar volume than EG, and therefore substitution of EG with BPEF decreases concentration of DMHN. The almost constant $\Delta n_{0}$ value implies that anisotropy of DMHN, BPEF and EG is almost the same. Thus, the decrease of $C_{\mathrm{R}}$ with increasing BPEF is due to the increase of flexibility rather than to the decrease of the anisotropy of the structural unit.

For PET(TF0), TF40 and TF70 with DMT as acid (TF series), $\Delta n_{0}$ decreases with increasing BPEF (See Table II). This result contrasts with the case of DMHN $=100$ mol\% in which $\Delta n_{0}$ was almost constant. Relatively large decrease of $\Delta n_{0}$ for TF series can be attributed to dilution of DMT, which is the most anisotropic unit. On the other hand, $M_{\mathrm{s}}$ increases with increasing BPEF for TF series. Direction of $M_{\mathrm{s}}$ variation with BPEF is opposite to HNF series. The increase of BPEF does not always enhance the flexibility. This is quite a reasonable result because flexibility is determined by the interaction between the consequent units in the chain. 
a)

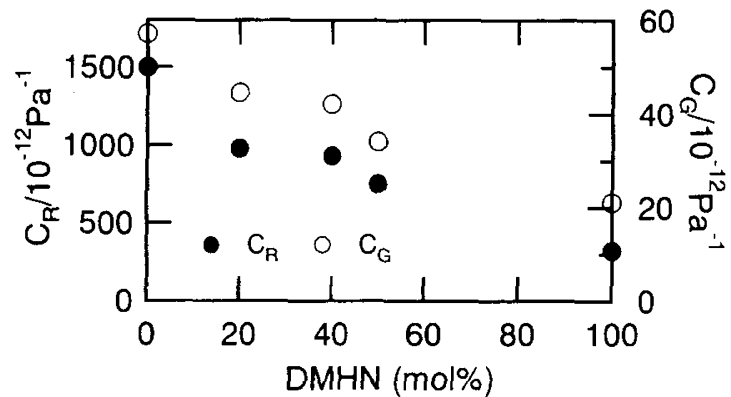

b)

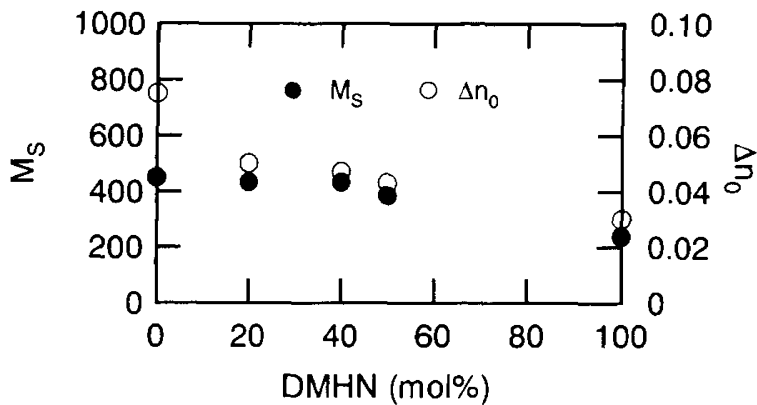

Figure 4. DMHN fraction dependence of a) stress-optical coefficients and b) $M_{\mathrm{s}}$ and $\Delta n_{0}$.

Two Measures of Birefringence, $C_{R}$ and $\Delta n_{0}$

The remaining birefringence is associated with the birefringence at a certain level of stress in flow and stress relaxation in the processing and may be represented by the stress-optical coefficient, $C_{\mathrm{R}}$. $C_{\mathrm{R}}$ characterizes anisotropy of segments, and therefore it depends on not only anisotropy of the structural units but also the size of segment. On the other hand, the intrinsic birefringence, $\Delta n_{0}$, represents the birefringence for oriented materials, e.g., for cross-linked polymers in elongation. The anisotropy of the structural unit apart from the chain flexibility may be represented by $\Delta n_{0}$. For molecular design of polymers with low birefringence, with regard to the remaining birefringence, one has to take two factors in consideration ; the anisotropy of the structural units and the chain flexibility. One known method for the former may be the use of saturated ring structure in the main chain. For the estimation of the chain flexibility, simple calculations such as that for the RIM model may be utilized.

\section{CONCLUSION}

Dynamic viscoelasticity and dynamic birefringence were investigated for a series of polyesters with various combinations of DMHN and DMT as acid component and BPEF and EG as diol component. The lowering of stress-optical coefficient was achieved by the displacement of phenylene (DMT) with a hydrogenated naphthylene ring (DMHN) in the main chain and by the introduction of BPFE. The former choice lowered the intrinsic birefringence but did not affect the chain flexibility. The latter enhanced the flexibility but did not affect a)

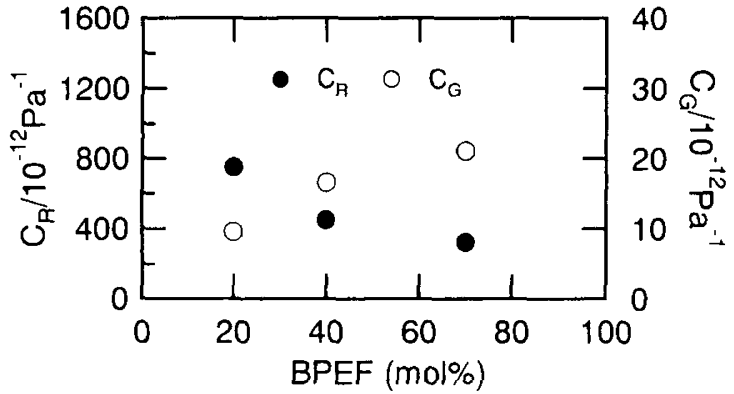

b)

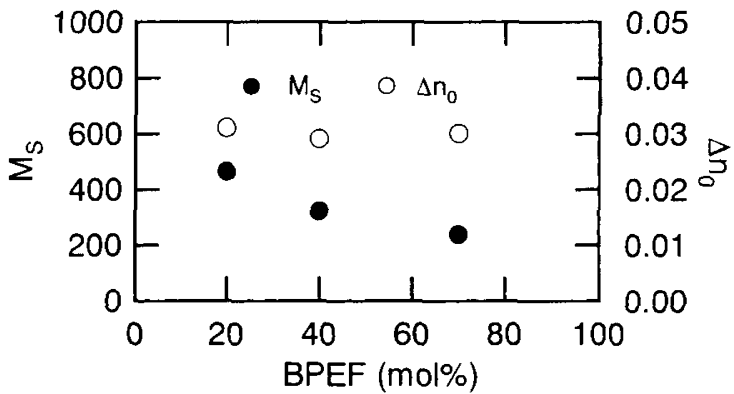

Figure 5. BPEF fraction dependence of a) stress-optical coefficients and b) $M_{\mathrm{s}}$ and $\Delta n_{0}$.

the intrinsic birefringence. The systematic result clearly revealed the importance of two different factors in molecular designs for polymers with low stress-optical coefficient.

Acknowledgment. This study was supported by the Grant-in-Aid for Scientific Research (No. 09450361) from the Ministry of Education, Science, Sports and Culture of Japan.

\section{REFERENCES}

1. K. Osaki, H. Okamoto, T. Inoue, and E.-J. Hwang, Macromolecules, 28, 3625 (1995).

2. T. Inoue and K. Osaki, Polym. J., 28, 76 (1996).

3. T. Inoue, H. Okamoto, Y. Mizukami, H. Matsui, H. Watanabe, T. Kanaya, and K. Osaki, Macromolecules, 29, 6240 (1996).

4. T. Inoue, H. Matsui, S. Murakami, S. Kojiya, and K. Osaki, Polymer, 38, 1215 (1997).

5. T. Inoue, O. Takiguchi, K. Osaki, T. Kohara, and T. Natsuume, Polym. J., 26, 133 (1994).

6. T. Inoue, H. Okamoto, K. Osaki, T. Kohara, and T. Natsuume, Polym. J., 27, 943 (1995).

7. T. Inoue and T. Saito, Func. Mat., 3, 21 (1987).

8. S. Iwata, E. Tsukahara, E. Nihei, and Y. Koike, Polym. Prepr., Jpn, 43, 2414 (1994).

9. K. Sakurai and M. Fuji, Polym. J., to be submitted.

10. T. Inoue, H. Okamoto, and K. Osaki, Macromolecules, 24, 5670 (1991).

11. T. Inoue and K. Osaki, Macromolecules, 29, 1595 (1996)

12. T. Inoue, D. S. Ryu, K. Osaki, and T. Takebe, J. Polym. Sci. Polym. Phys., 37, 399 (1999).

13. J. D. Ferry, "Viscoelastic Properties of Polymers", Wiley, New York, N.Y., 1980. 